EXTENDED REPORT

\title{
Molecular pathology of $X$ linked retinoschisis: mutations interfere with retinoschisin secretion and oligomerisation
}

\author{
T Wang, A Zhou, C T Waters, E O'Connor, R J Read, D Trump
}

Br J Ophthalmol 2006;90:81-86. doi: 10.1136/bjo.2005.078048

See end of article for authors' affiliations

.....................

Correspondence to: Professor Dorothy Trump, Academic Unit of Medical Genetics, University of Manchester, St Mary's Hospital, Manchester M13 OJH, UK; dorothy.trump@ manchester.ac.uk

Accepted for publication 4 August 2005

\begin{abstract}
Background/aim: X linked retinoschisis (XLRS) is caused by mutations in RS1 which encodes the discoidin domain protein retinoschisin, secreted by photoreceptors and bipolar cells. Missense mutations occur throughout the gene and some of these are known to interfere with protein secretion. This study was designed to investigate the functional consequences of missense mutations at different locations in retinoschisin.

Methods and results: The authors developed a structural model of the retinoschisin discoidin domain and used this to predict the effects of missense mutations. They expressed disease associated mutations and found that those affecting conserved residues prevented retinoschisin secretion. Most of the remaining mutations cluster within a series of loops on the surface of the $\beta$ barrel structure and do not interfere with secretion, suggesting this region may be a ligand binding site. They also demonstrated that wild type retinoschisin octamerises and associates with the cell surface. A subgroup of secreted mutations reduce oligomerisation (C59S, C219G, C223R).

Conclusions: It is suggested that there are three different molecular mechanisms which lead to XLRS: mutations interfering with secretion, mutations interfering with oligomerisation, and mutations that allow secretion and oligomerisation but interfere with retinoschisin function. The authors conclude that binding of oligomerised retinoschisin at the cell surface is important in its presumed role in cell adhesion.
\end{abstract}

$\mathrm{X}$ linked recessive juvenile retinoschisis is the leading cause of juvenile macular degeneration in males and is characterised by schisis within the retina, leading to visual deterioration. ${ }^{1-3}$ Affected males have reduced b-waves but normal a-waves on electroretinogram indicating an inner retinal abnormality. ${ }^{4}$

A large number of disease causing mutations in the gene RSI are recognised ${ }^{6}$ (www.dmd.nl/rs/rshome.html), the majority being missense substitutions. RSI encodes retinoschisin, a protein of unknown function secreted from photoreceptor and bipolar cells ${ }^{78}$ and contains a discoidin domain. ${ }^{9}$ Discoidin domains are implicated in cell adhesion or cell signalling. ${ }^{10}{ }^{11}$ The $R s i h$ knockout RS mouse has a retinal phenotype closely resembling the human disease with a generalised disruption of retinal cell layers. ${ }^{12}$ Replacement of the $R S 1$ gene leads to an improvement in retinal function and morphology, ${ }^{13}{ }^{14}$ supporting a role for retinoschisin in retinal architecture development through mediating interactions between cells and extracellular matrix.

RS mutations cluster within the discoidin domain. We have previously shown that many mutations lead to intracellular retention and degradation of mutant protein. ${ }^{15}$ Recent work indicates that retinoschisin functions as an octamer. ${ }^{16}{ }^{17}$

We describe work confirming and extending these data. We present our own structural model of the retinoschisis discoidin domain and interpret disease mutations with respect to the model. We describe data confirming retinoschisin octamerisation and cell surface association and our expression of mutations ${ }^{15}$ ). We use our data and those of others ${ }^{16}{ }^{17}$ to discuss potential molecular mechanisms leading to the disease.

\section{MATERIAL AND METHODS}

A homology based model of the retinoschisin discoidin domain was constructed with 3D-PSSM ${ }^{18}$ webserver (www.sbg.bio.ic.ac.uk/ 3dpssm) using as a scaffold the structure of the factor Va C2 domain, ${ }^{19}$ entry 1CZS in the $\mathrm{PDB}^{20}$

\section{Construction of mutant RS 1 by site directed mutagenesis}

Constructs of RSI mutations were generated by site directed mutagenesis using QuikChange (Stratagene) as described, ${ }^{15}$ genbank:cDNA NM 000330, protein NP 000321.

\section{Cell culture and transfection}

COS-7 cells were grown and transfected ${ }^{15} ; 72$ hours after transfection, cells and culture medium were collected. Stable cell lines expressing wild type and mutant proteins (C56S, R141H and F108C) were selected by G148.

\section{Western blot analysis}

Whole cell lysates were prepared and blotted. ${ }^{15}$ For nonreducing SDS-PAGE, samples were denatured in Laemmli buffer (room temperature 30 minutes) with $20 \mathrm{mM} \mathrm{N}$ ethylmaleimide (NEM) but without $\beta$-mercaptoethanol.

\section{Gel filtration analysis}

Gel filtration analysis was performed on a Superdex 200 column at room temperature after equilibration with $20 \mathrm{mM}$ TRIS-HCl, $0.15 \mathrm{M} \mathrm{NaCl}, 1 \mathrm{mM}$ EDTA, pH 7.4, a flow rate of $0.5 \mathrm{ml} / \mathrm{min}$, calibrated by molecular weight markers. Cell culture medium was concentrated to $1 \mathrm{ml}$ by Vivaspin (Vivascience) and applied onto the gel filtration column. Fifty fractions were collected, with $1.5 \mathrm{ml}$ per fraction.

\section{Cell surface biotinylation}

Cells were incubated with $0.5 \mathrm{mg} / \mathrm{ml}$ sulfo-NHS-LC-biotin in phosphate buffered saline (PBS) at $4^{\circ} \mathrm{C}$ for 30 minutes, washed with cold PBS containing $100 \mathrm{mM}$ glycine, lysed with RIPA buffer. A volume of $150 \mu \mathrm{l}$ of total cell lysate fraction

Abbreviations: NEM, N-ethylmaleimide; XLRS, $X$ linked retinoschisis 
A
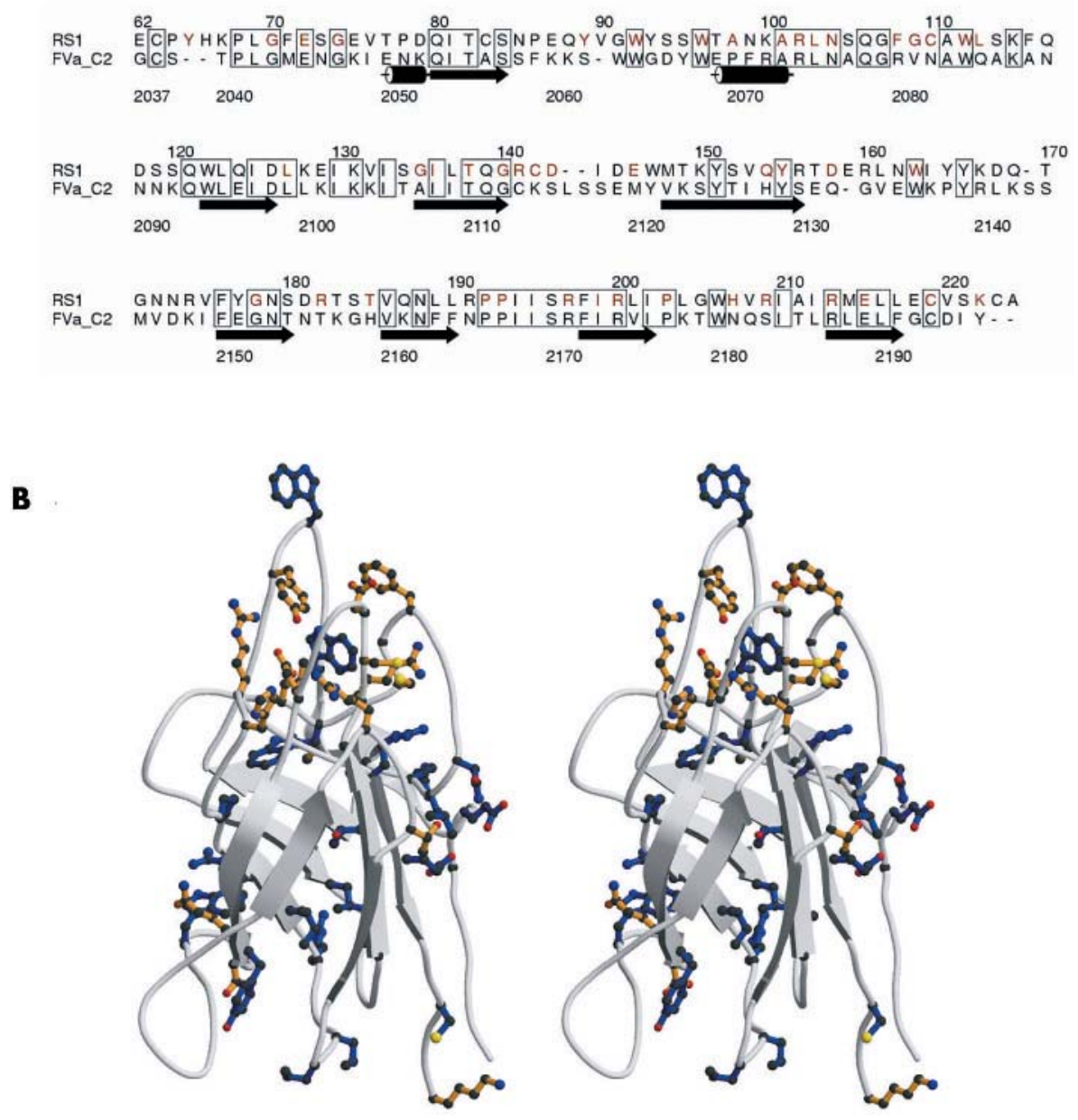

Figure 1 Homology based modelling of discoidin domain of retinoschisin. (A) Alignment of amino acid sequences of discoidin domains of retinoschisin (RS1) and factor Va (FVa_C2), prepared with Alscript. Boxes outline residues that are identical in the two sequences, retinoschisin residues mutated in disease are highlighted in red, and the secondary structure of the factor Va crystal structure ${ }^{19}$ indicated schematically. (B) Ribbon diagram (as stereo pair) of the homology based model of the retinoschisin discoidin domain showing the $\beta$ barrel structure with long loops, prepared with Molscript (www.avatar.se/molscript). The side chains shown indicate the positions of all mutations in retinoschisin patients to date. Sites of mutation that are conserved in the sequence alignment with factor $V a$ are shown in blue, while mutation sites that are not conserved are shown in orange.

was incubated with equal volumes of streptavidin-sepharose overnight, spun at $15000 \mathrm{~g}$ at $4^{\circ} \mathrm{C}$ for 15 minutes (supernatant $=$ intracellular fraction). Pelleted streptavidin beads were washed with RIPA buffer and biotinylated cell surface proteins eluted by heating in $150 \mu \mathrm{l}$ Laemmli buffer at $100^{\circ} \mathrm{C}$ for 5 minutes. Samples of cell fractions were subjected to SDS-PAGE followed by western blot. Calnexin antibody (Santa Cruz) 1:500 dilution was used to detect calnexin as an intracellular control.

\section{RESULTS}

The C2 domain of factor $\mathrm{Va}^{19}$ was chosen as the scaffold for homology modelling of the retinoschisin discoidin domain because it is the most closely related protein of known structure, ( $37 \%$ sequence identity). The goal was to construct a model showing the location of disease associated mutations within the overall fold. After we completed this modelling, similar homology based molecular models were published by two other groups. ${ }^{1621}$

The model was examined using computer graphics, highlighting residues substituted in RS missense mutations. A number of these residues are identical in the aligned sequences of retinoschisin and factor Va (figlA); the majority of these are buried in the hydrophobic core (fig 1B), so one would predict that mutations would destabilise the structure leading to intracellular retention as we have previously shown. ${ }^{15}$ Strikingly, most of the remaining (unconserved) mutations cluster within a series of loops on one end of the discoidin domain, comprising residues 85-96, 102-121, 141147, 181-185, and 203-212 (fig 1B). The clustering on the surface suggested that this region may be a ligand binding site.

\section{Analysis of the secretion profile of mutant retinoschisin}

We expressed 24 mutant forms of retinoschisin, seven of which had been published previously ${ }^{15}$ and analysed the medium for secretion (table 1 and fig 2). The mutations encompassed those altering conserved and non-conserved residues within the discoidin domain, those affecting cysteine residues throughout the molecule, and one signal peptide mutation. 


\begin{tabular}{|c|c|c|c|c|c|}
\hline Mutations & Base changes & $\begin{array}{l}\text { Amino acid } \\
\text { changes }\end{array}$ & Secretion & $\begin{array}{l}\text { Mutation affects } \\
\text { conserved residue }\end{array}$ & $\begin{array}{l}\text { Discoidin } \\
\text { domain }\end{array}$ \\
\hline $\mathrm{L} 12 \mathrm{H}^{*}$ & GGG-GAG & Leu-His & - & - & No \\
\hline C59S* & TGT-AGT & Cys-Ser & + & - & No \\
\hline G70S* & TGT-AGT & Gly-Ser & - & + & Yes \\
\hline Y89C & TAT-TGT & Tyr-Cys & - & - & Yes \\
\hline W92C & TGG-TGC & Trp-Cys & - & + & Yes \\
\hline W96R & TGG-CGG & Trp-Arg & - & + & Yes \\
\hline $\mathrm{R} 102 \mathrm{~W}^{*}$ & CGG-TGG & Arg-Trp & - & + & Yes \\
\hline N104K & AAC-AAG & Asn-Lys & - & + & Yes \\
\hline F108C & TाT-TGT & Phe-Cys & + & - & Yes \\
\hline G109R* & GGG-CGG & Gly-Arg & - & - & Yes \\
\hline G109E & GGG-GAG & Gly-Glu & - & - & Yes \\
\hline $\mathrm{Cl} 10 \mathrm{Y}$ & TGT-TAT & Cys-Tyr & - & - & Yes \\
\hline W112C & TGG-TGT & Trp-Cys & - & + & Yes \\
\hline L113F & СТC-TTC & Leu-Phe & - & - & Yes \\
\hline $\mathrm{R} 141 \mathrm{H}$ & CGC-CAC & Arg-His & + & - & Yes \\
\hline $\mathrm{R} 141 \mathrm{G}^{*}$ & CGC-GGC & Arg-Gly & + & - & Yes \\
\hline $\mathrm{Cl} 42 \mathrm{~W}^{*}$ & TGT-TGG & Cys-Trp & - & + & Yes \\
\hline Dl $43 \mathrm{~V}$ & GAC-GTC & Asp-Val & - & - & Yes \\
\hline R182C & CGC-TGC & Arg-Cys & + & - & Yes \\
\hline H207Q & CAC-CAG & His-Gln & + & - & Yes \\
\hline $\mathrm{R} 209 \mathrm{H}$ & CGC-CAC & Arg-His & + & - & Yes \\
\hline R213W & CGG-TGG & Arg-Trp & - & + & Yes \\
\hline C219G & TGC-GGC & Cys-Gly & + & + & Yes \\
\hline C223R & TGT-CGT & Cys-Arg & + & - & No \\
\hline
\end{tabular}

Three mutations (L12H, C59S, and C223R) are outside the discoidin domain and one $(\mathrm{Ll} 2 \mathrm{H})$ inhibits cleavage of the signal peptide. ${ }^{15}$ We confirmed that $\mathrm{C} 59 \mathrm{~S}^{15}$ and C223R did not interfere with secretion. ${ }^{16}$ Of the nine mutations affecting conserved residues, one, C219G, was successfully secreted while eight resulted in intracellular retention (table 1 and fig 2). Twelve mutations affected non-conserved residues, of which six (F108C, R141H, R141G, R182C, H207Q, R209H) were secreted. These are predicted to cluster at the loop region (fig 2B). Y89 and W92, also found in the loop region, were mutated to cysteine, so their failure to be secreted may be the result of aggregation through the formation of inappropriate disulfide bridges.

\section{Retinoschisin associates with the cell surface}

We investigated whether wild type retinoschisin interacts with the cell surface (fig 3). We biotin labelled cell surface proteins and captured these by streptavidin coated sepharose beads. Retinoschisin was present in the cell surface fraction, cell lysate, and medium indicating it is associated with the cell membrane after secretion unlike the intracellular control calnexin. As retinoschisin is a secretory protein this suggests that anchoring to the cell membrane is through interaction with other molecules.

\section{Octamerisation of retinoschisin}

Retinoschisin is predicted to form oligomers. ${ }^{716}$ We investigated this for wild type retinoschisin and the secreted mutants using a combination of non-reducing PAGE (fig 4A) and gel filtration (fig 4B). The results confirm that retinoschisin does form oligomers (MW approximately $250 \mathrm{kDa}$ ) and that three of the cysteine substitution mutants (C59S, C219G, and C223R) fail to form oligomers of the same size.

The majority of wild type retinoschisin in Weri-Rbl cells, the stable transfected COS-7 cell line and R141H was eluted in fractions 17-19. The estimated molecular weight of these fractions was surprisingly low (approximately $66 \mathrm{kDa}$ ) but re-analysis by non-reducing PAGE revealed the band had the same mobility as the large molecular weight band described above. Retinoschisin was consistently eluted in later fractions than expected which may indicate the protein is adhering to the gel matrix. For C59S most of the protein was eluted later still indicating an interference with full oligomerisation. F108C was eluted earlier indicating larger oligomers than both wild type and other mutants tested. This was confirmed by the re-analysis of the peak fractions by non-reducing PAGE (fig 4C). Interestingly, in the Weri-Rbl cells some wild type retinoschisin was eluted in earlier fractions 3-7 suggesting that some retinoschisin may be associating with another protein within the Weri-Rbl medium not present in the COS-7 medium.

We noted that heating interfered with the stability of the oligomer. We investigated the effects of heating and treatment with the sulfhydryl modifying agent NEM (fig 4D) and confirmed this was due to interference of free cysteine residues with disulphide bonds. When the sample (fraction 19) was treated with NEM before heating a single high molecular weight band was observed (fig 4D) indicating the modification of the sulfhydryl groups of the free cysteine residues prevented their competing for disulphide bond formation. Heating in the absence of NEM reveals a laddering of eight bands confirming octamerisation.

\section{DISCUSSION}

Mutations in retinoschisin lead to X linked retinoschisis, ${ }^{6}{ }^{9}$ which causes retinal splitting. ${ }^{22}$ In addition, the knockout mouse model has a generalised disruption of retinal layers, ${ }^{12}$ suggesting that retinoschisin, like other discoidin domain proteins, has a role in cell adhesion and is involved in controlling the development and maintenance of retinal architecture.

The retinoschisin discoidin domain has strong homology to discoidin domains of other proteins, which allowed us to generate a model showing the location of disease associated mutations. Our model, derived separately from those of other groups, ${ }^{16}{ }^{21}$ shows a similar structure and allowed us to identify for the first time the position of disease associated missense mutations within the model. This enabled us to correlate the effects of mutation position with successful secretion from cells. A subset of missense mutations did not interfere with secretion and the majority of these affected surface residues within the loop region (F108C, R141H, R141G, R182C, H207Q, and R209H), suggesting a functional 


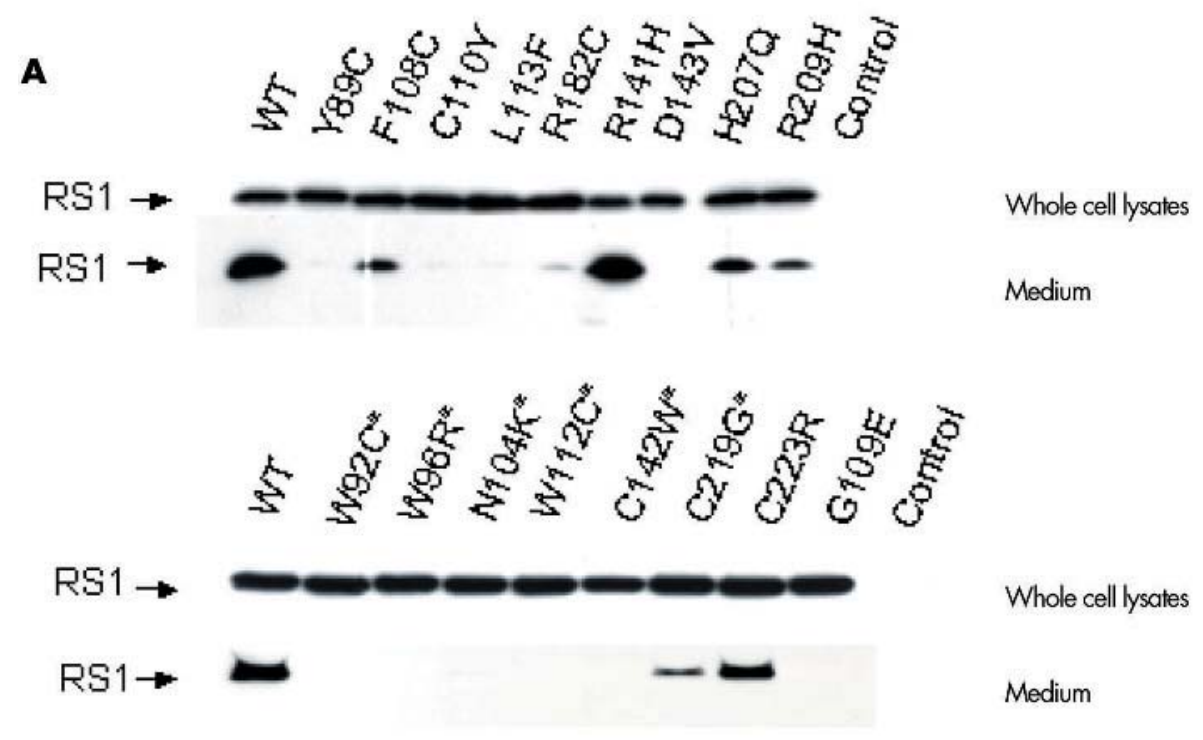

B
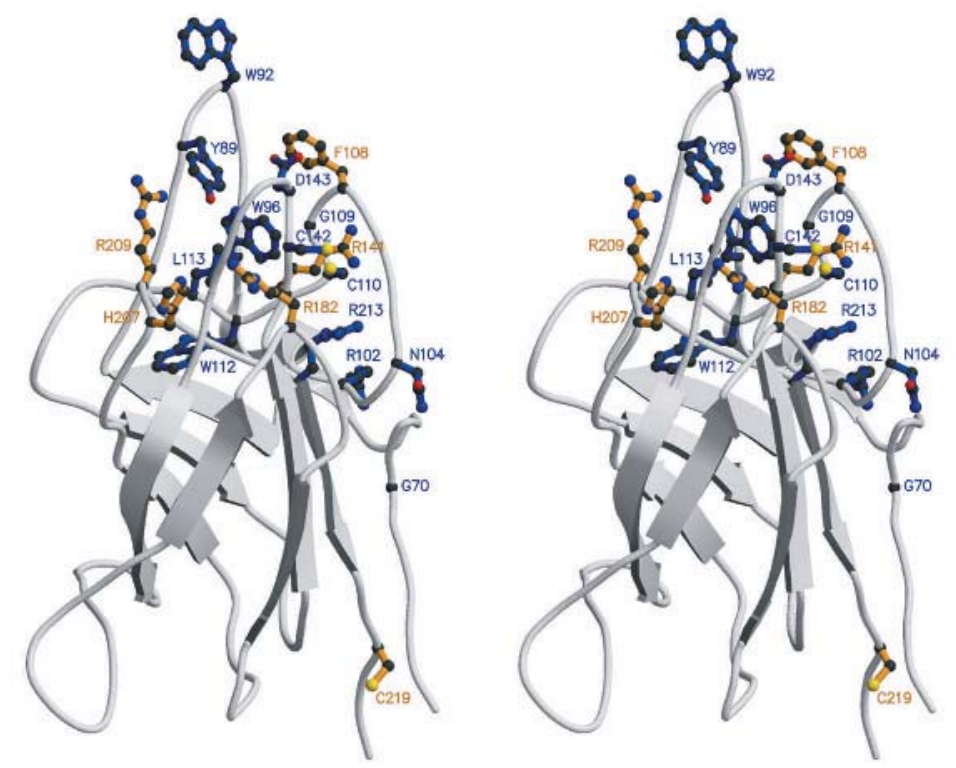

Figure 2 Western blot of expressed mutant retinoschisin. (A) Whole cell lysates and cell culture medium of wild type and mutant retinoschisin were subjected onto SDS-PAGE and immunoblotted using anti-retinoschisin antibody. *Indicates mutations at conserved residues in discoidin domain. "Control" indicates transfection with pcDNA 3.1 empty vectors only. Six of the 12 mutations affecting non-conserved residues and two mutations affecting the cysteines outside the discoidin domain (C59 and C223) were secreted. (B) Ribbon diagram (as stereo pair) showing side chains for residues investigated. Sites of mutation that interfered with secretion of the mutant protein are shown in blue, while mutation sites that did not interfere with secretion are shown in light orange.

rather than structural importance for this region. This provides both theoretical and experimental evidence supporting existence of a functional binding pocket lying closely linked to a hydrophobic surface region.

$\mathrm{Wu}$ and Molday have published elegant data describing the octamerisation of retinoschisin and the underlying disulphide bonding. ${ }^{17}$ They expressed mutant forms of retinoschisin and interestingly a comparison of their published data and our data indicates that different mutations affecting the same residue have a different consequencethat is, Rl4lG and Rl41H are both secreted (data shown here) but R141C is not. ${ }^{17}$ This suggests that the addition of this cysteine may lead to the formation of abnormal disulphide bridges giving intracellular aggregation. In addition, our results differed for one mutation, R182C, for which we detected secretion at a low level and could concentrate this to a similar level to other secreted protein, whereas Wu et al failed to detect secretion. This may be because of differences in methodology. F108C showed an increase in oligomerisation. We hypothesise that this additional cysteine residue is available for disulphide bond formation and leads to aggregation. 

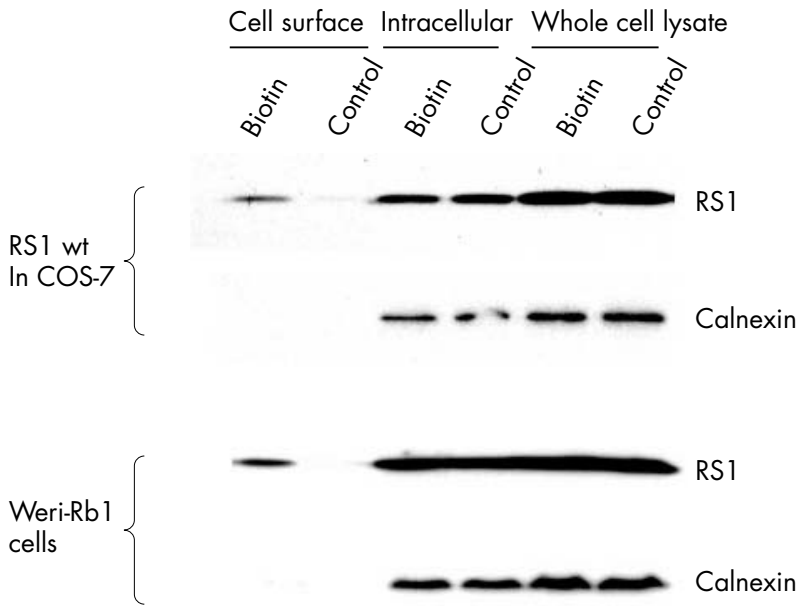

Figure 3 Biotinylation of cell surface proteins. Following biotinylation cells were lysed and fractionated. Control cells were not exposed to biotin but otherwise followed the same protocol. Western blot analysis was used to detect the presence of retinoschisin, or the intracellular control protein (calnexin). Retinoschisin was detected in all fractions including the cell surface in the biotinylated cells but not on the cell surface of the control cells. Calnexin was not detected in the cell surface fractions.

Reviewing our own data and those of Wu et al we suggest three mechanisms of disease causation.

(1) Mutations interfering with retinoschisis secretion

(2) Mutations interfering with retinoschisin octamerisation

(3) Mutations that allow secretion and octamerisation but interfere with protein function.

We have considered disease expression for patients with mutations in each of these groups and have found no correlation between the molecular mechanism and severity. ${ }^{23}$ This suggests there may be other factors influencing disease severity such as genetic modifiers or environmental influences. Recent work describing the successful treatment of knockout mice with targeted gene therapy ${ }^{13}{ }^{14}$ indicates this may be a possible future therapy for patients. Since women who are heterozygous for the mutant gene (and therefore express both mutant and wild type retinoschisin) do not have retinal pathology, this suggests there is unlikely to be a detrimental interaction between the patient's own mutant retinoschisin and retinoschisin delivered from gene therapy. As we cannot predict disease severity such treatment would have to be considered on an individual basis. Molecular genetic testing in XLRS has facilitated diagnosis and improved genetic counselling for families. However, our current understanding of XLRS molecular pathology is still limited and further comprehensive analysis of retinoschisin and potential binding partners is important.

\section{ACKNOWLEDGEMENTS}

We are grateful to Bernard Weber (Universitsät Wurzburg, Germany) for providing the RSI cDNA clone and to Graeme Black (Manchester University) for helpful advice and comments on the manuscript. This study was funded by the Medical Research Council UK. RJR is supported by a Principal Research Fellowship from the Wellcome Trust.

\section{Authors' affiliations}

T Wang, E O'Connor, D Trump, Academic Unit of Medical Genetics, School of Medicine, University of Manchester and Centre for Molecular Medicine, Faculty of Medical and Health Sciences, University of Manchester, Manchester M13 OJH, UK

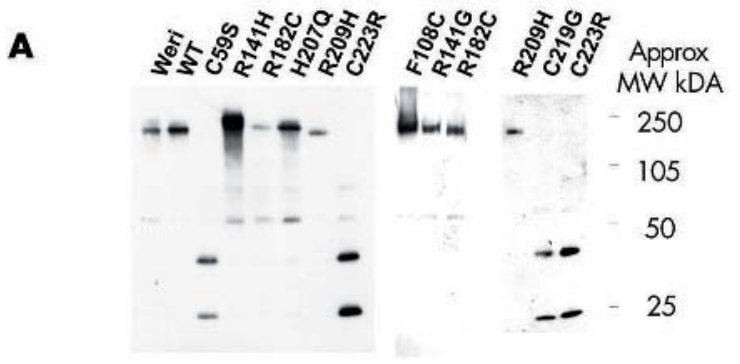

B

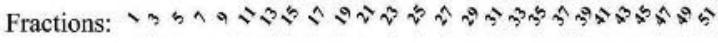
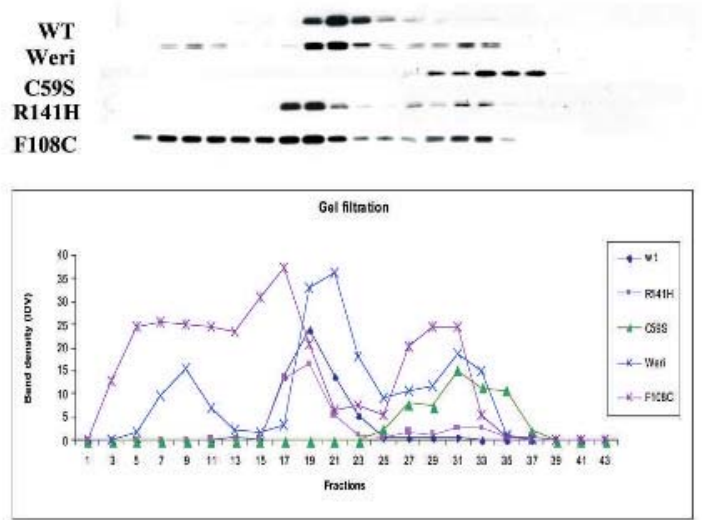

C

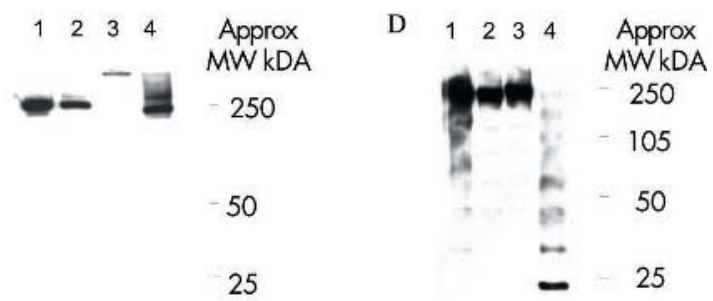

Figure 4 Non-reducing gel and gel filtration for secreted wild type (WT) retinoschisin, retinoschisin mutants, and Weri-Rbl Retinoschisin. (A) Non-reducing gel for WT and mutant retinoschisin showing oligomerisation of WT and mutants. Three of the mutants (C59S, C219G, and C223R) formed only monomers and dimers. (B) Gel filtration analysis using concentrated cell culture media from Weri-Rbl cells, stably transfected wild type RS1 and C59S, F108C and R141H mutants. 50 fractions were collected. The results of western blot analysis are shown from alternate fractions in the upper panel and indicate WT retinoschisin (and $\mathrm{R} 141 \mathrm{H}$ ) forms oligomers. C59S is detected in later fractions consistent with monomers, whereas F108C is eluted in earlier fractions indicating a greater tendency to oligomerisation than WT. The lower panel depicts the same data in graph form. (C) Non-reducing gel analysing fractions from gel filtration. Lanes 1 and 2 show fractions 19 from Weri-Rbl cells and COS-7 cells stably expressing WT retinoschisin respectively and lanes 3 and 4 show fractions 5 and 17 from COS-7 cells stably expressing F108C retinoschisin, respectively. F108C mutant retinoschisins from fraction 5 runs slower than the other fractions indicating a larger sized multimer. (D) Non-reducing gel demonstrating the effects of heating and NEM treatment on the WT retinoschisin. Each lane contains conditioned WT cell medium. Lane 1 unheated and untreated, lane 2 treated with NEM in $4 M$ urea at room temperature, lane 3 treated with NEM in $4 \mathrm{M}$ urea and heated to $100^{\circ} \mathrm{C}$ for 3 minutes, lane 4 heated to $100^{\circ} \mathrm{C}$ for 3 minutes without NEM or urea. Laddering indicating dissociation of the oligomer is seen in lane 4 only.

T Wang, C T Waters, E O'Connor, D Trump, Department of Medical Genetics and Cambridge Institute for Medical Research, University of Cambridge CB2 2XY, UK

A Zhou, R J Read, Department of Haematology and Cambridge Institute for Medical Research, University of Cambridge CB2 2XY, UK 


\section{REFERENCES}

1 George ND, Yates JR, Moore AT. X linked retinoschisis. Br J Ophthalmol 1995;79:697-702.

2 George ND, Yates JR, Moore AT. Clinical features in affected males with Xlinked retinoschisis. Arch Ophthalmol 1996;114:274-80.

3 Seiving PA. Juvenile retinoschisis. In: Traboulsi E, ed. Genetic diseases of the eye. New York: Oxford University Press, 1998:347-56.

4 Peachey NS, Fishman GA, Derlacki DJ, et al. Psychophysical and electroretinographic findings in X-linked juvenile retinoschisis. Arch Ophthalmol 1987; 105:513-16.

5 Bradshaw K, George N, Moore A, et al. Mutations of the XLRSI gene cause abnormalities of photoreceptor as well as inner retinal responses of the ERG. Doc Ophthalmol 1999;98:153-73.

6 The Retinoschisis Consortium, Functional implications of the spectrum of mutations found in 234 cases with X-linked juvenile retinoschisis (XLRS) Human Molecular Genetics 1998;7:1 185-92.

7 Grayson C, Reid SN, Ellis JA, et al. Retinoschisin, the X-linked retinoschisis protein, is a secreted photoreceptor protein, and is expressed and released by Weri-Rbl cells. Hum Mol Genet 2000;9:1873-9.

8 Molday LL, Hicks D, Saver CG, et al. Expression of X-linked retinoschisis protein RS1 in photoreceptor and bipolar cells. Invest Ophthalmol Vis Sci 2001;42:816-25.

9 Saver CG, Gehrig A, Warneke-Wittstock R, et al. Positional cloning of the gene associated with $\mathrm{X}$-linked juvenile retinoschisis. Nat Genet 1997:17:164-70.

10 Baumgartner S, Hofmann K, Chiquet-Ehrismann R, et al. The discoidin family revisited: New members from prokaryotes and a homology based fold prediction. Protein Sci 1998;7:1626-31.

11 Vogel W. Discoidin domain receptors: structural relations and functional implications. FASEB J 1999:13:S77-S82.

12 Weber BH, Schrewe H, Molday LL, et al. Inactivation of the murine X-linked juvenile retinoschisis gene, Rs $1 \mathrm{~h}$, suggests a role of retinoschisin in retinal cell ayer organization and synaptic structure. Proc Natl Acad Sci USA 2002;99:6222-7.
13 Zeng $Y$, Takada $Y$, Kiellstrom S, et al. RS-1 gene delivery to an adult Rs $1 \mathrm{~h}$ Knockout mouse model restores ERG b-wave with reversal of the electronegative waveform of X-linked retinoschisis. Invest Ophthalmol Vis Sci 2004;45:3279-85

14 Min SH, Molday LL, Seeliger MW, et al. Prolonged recovery of retinal structure/function after gene therapy in an Rs $1 \mathrm{~h}$-deficient mouse model of Xlinked juvenile retinoschisis. Mol Ther 2005

15 Wang T, Waters CT, Rothman AM, et al. Intracellular retention of mutant retinoschisin is the pathological mechanism underlying X-linked retinoschisis. Hum Mol Genet 2002; 11:3097-105.

16 Wu WW, Molday RS. Defective discoidin domain structure, subunit assembly, and endoplasmic reticulum processing of retinoschisin are primary mechanisms responsible for X-linked retinoschisis. J Biol Chem 2003;278:28139-46.

17 Wu WW, Wong JP, Kast J, et al. RS1, a discoidin domain-containing retinal cell adhesion protein associated with $\mathrm{X}$-linked retinoschisis, exists as a novel disulfide-linked octamer. J Biol Chem 2005;280:10721-30.

18 Kelley LA, MacCallum RM, Sternberg MJ. Enhanced genome annotation using structural profiles in the program 3D-PSSM. J Mol Biol 2000;299:499-520.

19 Macedo-Ribeiro S, Bode W, Huber R, et al. Crystal structures of the membrane-binding C2 domain of human coagulation factor V. Nature 1999:402:434-9

20 Berman HM, Westbrook J, Feng Z, et al. The Protein Data Bank. Nucleic Acids Res 2000;28:235-42.

21 Fraternali F, Cavallo L, Musco G. Effects of pathological mutations on the stability of a conserved amino acid triad in retinoschisin. FEBS Lett 2003;544:21-6.

22 Kirsch LS, Brownstein S, de Wolff-Rouendaal D. A histopathological, ultrastructural and immunohistochemical study of congenital hereditary retinoschisis. Can J Ophthalmol 1996:31:301-10.

23 Pimenides D, George NDL, Yates JRW, et al. X-linked retinoschisis: clinical phenotype and RS1 genotype in 86 UK patients. J Med Genet 2005; $42:$ e35. 